\title{
How Can Organizations Design Purposeful Human-AI Interactions: A Practical Perspective From Existing Use Cases and Interviews
}

\author{
Silvana Hinsen \\ University of Bayreuth, \\ Bayreuth, Germany \\ silvana.hinsen@, \\ uni-bayreuth.de
}

\author{
Peter Hofmann \\ Project Group BISE of the \\ Fraunhofer FIT, \\ Bayreuth, Germany \\ peter.hofmann@, \\ fit.fraunhofer.de
}

\author{
Jan Jöhnk \\ FIM Research Center, \\ Bayreuth, Germany \\ jan.joehnk@,fim-rc.de
}

\author{
Nils Urbach \\ Project Group BISE of the \\ Fraunhofer FIT, \\ Frankfurt University of \\ Applied Sciences, \\ Frankfurt, Germany \\ nils.urbach@fim-rc.de
}

\begin{abstract}
Artificial intelligence (AI) currently makes a tangible impact in many industries and humans' daily lives. With humans interacting with AI agents more regularly, there is a need to examine human-AI interactions to design them purposefully. Thus, we draw on existing AI use cases and perceptions of human-AI interactions from 25 interviews with practitioners to elaborate on these interactions. From this practical lens on existing human-AI interactions, we introduce nine characteristic dimensions to describe human-AI interactions and distinguish five interaction types according to AI agents' characteristics in the human-AI interaction. Besides, we provide initial design guidelines to stimulate both research and practice in creating purposeful designs for human-AI interactions.
\end{abstract}

\section{Introduction and foundations}

Companies are finding themselves in a veritable run on artificial intelligence (AI) and its business potentials. As a general-purpose technology, scholars and practitioner experts predict that AI applications will disrupt industries and work in organizations [1]. While AI has been an on-and-off topic of debate for decades, its recent breakthrough is fueled, among others, by easily accessible computational resources, the abundance and accessibility of data, and advances in machine learning models [2]. Thus, AI technologies not only permeate product and service portfolios but also enable business model innovations.

While usability and user behavior in adopting digital technologies has spurred notable research, the AI frontier raises more fundamental issues. For one thing, machine learning (as the currently most dominant technological approach to $\mathrm{AI}$ ) relies on data to train and test its models that perceive, extract, structure and classify, predict, or generate outcomes in the real world [2]. The path towards these outcomes often resembles a 'black box' that humans can hardly understand or interpret. For another thing, AI applications enable a continuum from automating to augmenting human tasks [3]. This results in a plethora of potential interaction scenarios between humans and AI applications.

With AI applications increasingly moving into human tasks and interactions, we must reconsider how people use, apply, and interact with digital technologies. An AI (or intelligent) agent is a (more or less) perceptible actor in previously human tasks and teams. When interacting with AI agents, users often assign them human and social characteristics [4]. Accordingly, humans perceive a social presence by AI agents so that social behavior resembles human-human interaction [5]. Considering this, we expect a different logic of how we interact with $\mathrm{AI}$ agents and integrate them into our private and professional lives [6]. For instance, in 2018, Google Duplex surprised observers with a personal AI assistant capable of making reservations that were virtually indistinguishable from a human caller. At the same time, badly designed human-AI (H-AI) interactions can impair users' and customers' acceptance and trust in companies' offerings [7, 8]. For instance, Google Duplex raised concerns that its indistinguishability from human calls is problematic because it feigns human interactions [9].

Application scenarios for AI agents span many domains including scenarios where they co-exist with humans and closely interact with each other in hybrid systems [3, 10]. Despite the rise of these close interactions, research on how to structure and design $\mathrm{H}$ $\mathrm{AI}$ interactions is still scarce $[8,11,12]$. In this paper, we seek to stimulate further research in this regard by providing a practical perspective on this matter and elucidating how we may interact with a technology that 
has cognitive traits and capabilities [4]. Thus, we ask: How can we structure human-AI interactions in existing and potentially conceivable AI applications?

Interactions between humans and AI agents are manifold and can take place in a wide range of applications, from virtual assistants who provide people with weather forecasts based on location and weather data to industrial robots as intelligent employees that support production processes. To contribute to a further discussion of H-AI interactions' diversity and complexity, we followed an exploratory research approach by conducting expert interviews.

The concept of H-AI interaction has evolved and is continually changing owing to new opportunities and properties of interaction [4]. Historically, research and practice first discussed traditional human-human interactions, characterized by social and emotional aspects. Different disciplines of the social and communication sciences and psychology have defined the basic concepts of these interactions $[13,14]$. In the course of technological progress, the concept of interaction has been extended to include the disciplines of human-computer, human-machine, and human-robot interaction [15-19]. These established disciplines and the understanding of human interaction with the digital agency they entail provide valuable insights for $\mathrm{H}-\mathrm{AI}$ interactions. As organizations increasingly adopt AIenabled systems, ergonomic aspects in the sense of usercentric design become even more important [20].

However, critical aspects of human interaction with technology were already a topic in research into humancomputer and human-robot interactions [16, 19]. For instance, occupational safety requirements are a top priority in the design of human-robot interactions in industrial production environments. Even though the importance of interaction design is known, prior work falls short to focus on the design of $\mathrm{H}-\mathrm{AI}$ interaction to increase acceptance and trust towards AI agents. [8] Consequently, users' expectations towards AI agents' social cues, which trigger humans' social responses, are often not met in H-AI interactions. However, the aspect of social responses drives the complexity of H-AI interactions, especially considering its large number of potential application scenarios as well as its roles in tasks and interactions that were previously reserved for humans.

We seek to contribute to the discourse of $\mathrm{H}-\mathrm{AI}$ interactions by three means: First, we define nine interaction dimensions to describe the course of $\mathrm{H}-\mathrm{AI}$ interactions. Second, since research still lacks an understanding of the role AI agents can take on in interactions [8], we contribute to the body of knowledge by deriving five interaction types. Lastly, we define 12 design guidelines to address the influencing factors users' expectations, anthropomorphism, transparency, and personalization on the acceptance and trust of humans in H-AI interactions. Thereby, we provide an improved understanding of the interaction course and the roles an AI agent can mimic. To summarize, our paper facilitates H-AI interactions that fulfill their intended purpose in a way that builds acceptance and trust in humans, i.e. enabling the purposeful design of $\mathrm{H}-\mathrm{AI}$ interaction.

\section{Research approach}

Our research approach in this study was threefold. First, to provide a preliminary conceptual foundation for the practice-driven analysis, we collected literature from various disciplines (e.g., psychology, communication studies, human-computer interaction) to better understand interactions (with technology) and their constitutive elements. Following a scoping study approach [21], we drew on work on interactions between humans and other humans, between humans and computers, humans and machines, and humans and robots as relevant groundwork for H-AI interactions' specifics (see Section 1). By searching the online database Google Scholar, this approach primarily sought to rapidly map the fields of study and derive a

Table 1. Overview of interviewed experts

\begin{tabular}{|c|c|c|c|c|}
\hline ID & Interviewee position & Industry & $\begin{array}{c}\text { Company } \\
\text { size }\end{array}$ & $\begin{array}{l}\text { Intervieu } \\
\text { duration }\end{array}$ \\
\hline E01 & Senior Data Scientist & \multirow[b]{2}{*}{ Automotive } & \multirow[b]{2}{*}{$<100,000$} & \multirow[b]{2}{*}{$53 \mathrm{~min}$} \\
\hline E02 & $\begin{array}{c}\text { Director: Digital } \\
\text { Transformation }\end{array}$ & & & \\
\hline E03 & Chief Business Officer & IT & $<10$ & $58 \mathrm{~min}$ \\
\hline E04 & Managing Partner & Venture capital & $<50$ & $60 \mathrm{~min}$ \\
\hline E05 & $\begin{array}{l}\text { IT Architect Enterprise } \\
\text { Operations Center and } \\
\text { Workload Automation }\end{array}$ & IT & $>100,000$ & $61 \mathrm{~min}$ \\
\hline E06 & $\begin{array}{c}\text { Professor of Innovation and } \\
\text { Technology Management }\end{array}$ & Research & $<10$ & $50 \mathrm{~min}$ \\
\hline E07 & $\begin{array}{l}\text { Head: Asset Intelligence } \\
\text { Center }\end{array}$ & Logistics & $<50,000$ & $55 \mathrm{~min}$ \\
\hline E08 & Digital Advisor & Software & $>100,000$ & $70 \mathrm{~min}$ \\
\hline E09 & Senior Digital Expert & Manufacturing & $<25,000$ & $57 \mathrm{~min}$ \\
\hline E10 & $\begin{array}{l}\text { Head: Strategy and } \\
\text { Innovation }\end{array}$ & IT & $<50$ & $66 \mathrm{~min}$ \\
\hline E11 & Managing Director & IT & $<50$ & $52 \mathrm{~min}$ \\
\hline E12 & Member, Executive Board & Insurance & $<5,000$ & $49 \mathrm{~min}$ \\
\hline E13 & Head: Operations & Healthcare & $<50$ & $53 \mathrm{~min}$ \\
\hline E14 & Head: Functional Controlling & $\begin{array}{c}\text { Pharma and } \\
\text { agriculture }\end{array}$ & $>100,000$ & $56 \min$ \\
\hline E15 & Director & Automotive & $<50$ & $62 \mathrm{~min}$ \\
\hline E16 & $\begin{array}{c}\text { Vice President: Core } \\
\text { Business Apps } \\
\end{array}$ & Manufacturing & $<25,000$ & $56 \min$ \\
\hline E17 & CIO & Automotive & $>100.000$ & $79 \mathrm{~min}$ \\
\hline E18 & Head: IT & Construction & $<100,000$ & $54 \mathrm{~min}$ \\
\hline E19 & Head: Digital Unit & Manufacturing & $<5,000$ & $46 \mathrm{~min}$ \\
\hline E20 & $\mathrm{CIO} /$ Vice President IT & Automotive & $<100,000$ & $58 \mathrm{~min}$ \\
\hline E21 & CEO & IT & $<50$ & $58 \mathrm{~min}$ \\
\hline E22 & CEO & Automotive & $<50$ & $49 \mathrm{~min}$ \\
\hline E23 & $\mathrm{CEO}$ & Consulting & $<500$ & $53 \mathrm{~min}$ \\
\hline E24 & $\begin{array}{l}\text { Head: Center of Excellence } \\
\text { and IT Automation }\end{array}$ & Manufacturing & $<25,000$ & $58 \mathrm{~min}$ \\
\hline E25 & CEO & IT & $<250$ & $72 \mathrm{~min}$ \\
\hline
\end{tabular}


preliminary understanding of the course of interactions as preparation for the subsequent research steps [21].

In the second step, to reflect on potential specifics in H-AI interactions, we collected existing use cases of AI-enabled systems via an unstructured online search. Thereby, we engaged in joint discussion in the author team to contrast the preliminary understanding from extant work with practice-driven applications. For instance, we found that while existing research from other domains typically assumes a transparent interaction context (e.g., a user recognizes that s/he uses a computer), this must not always be the case for AI applications (e.g., Google Duplex). Other examples were the continuum from automating to augmenting tasks and the mutual dependency through potentially action-based learning of AI-enabled systems. Building on these revelations, we designed our exploratory interview study to gain a deeper practical perspective on the current implementation and perception of $\mathrm{H}-\mathrm{AI}$ interactions.

In the third step, we conducted 25 expert interviews to further explore AI applications' specifics [22]. The resulting insights formed our primary source of evidence and constitute the core foundation of our analysis. The experts (E) contribute perspectives from various industries, AI startups, and research (see Table 1). We approached experts via convenience sampling from our industry network and carefully selected them based on their involvement in the strategic design and application of AI agents in their companies. Incorporating the findings from the first two research steps, we used a semi-structured interview guideline with open questions to organize the interviews in three areas: a) understanding and development of AI, b) characteristic interaction dimensions and types, and c) influencing factors on $\mathrm{H}-\mathrm{AI}$ interactions. With experts' consent, we recorded and transcribed the interviews for later analysis. We used open coding to explore the data in the three question areas without imposing any category system [23]. Further, we revisited the material from the first research steps to compare it to our empirical data for consensuses, contradictions, and additions. After individual coding from one author, we jointly discussed the findings in the author team to abstract the findings across interviews and derive our conceptualization of $\mathrm{H}-\mathrm{AI}$ interactions.

\section{Conceptualizing human-AI interactions}

In this section, we present and explain the derived H-AI interaction dimensions and types. This section comprises our conceptual understanding from the exploratory expert interviews complemented by findings from the literature and the use cases. By describing the courses and contexts of H-AI interactions, the interaction dimensions help understand real-world examples of $\mathrm{H}-\mathrm{AI}$ interactions and derive $\mathrm{H}$ AI interaction types. Accordingly, H-AI interaction types are typical configurations of H-AI interaction dimensions. This section takes a use case perspective since the focus should not only be on the technology. "At the end of the day, AI is just a technology [...]. People do not see AI itself. [...] They have an application" (E15).

\subsection{Human-AI interaction dimensions}

We introduce nine interaction dimensions that describe H-AI interactions as a set of interdependent, consecutive, and directional actions (see Figure 1).

Interaction transparency refers to the degree of consciousness with which a person interacts with an AI agent. It can lie in a spectrum between perfect consciousness and perfect unconsciousness. This spectrum also includes interactions that can be classified as pre-conscious. In contrast to the two extremes, the latter interaction with $\mathrm{AI}$ agents does not take place directly consciously but can be identified as interaction with AI agents via cognitive considerations. One example of this is the passive monitoring of the

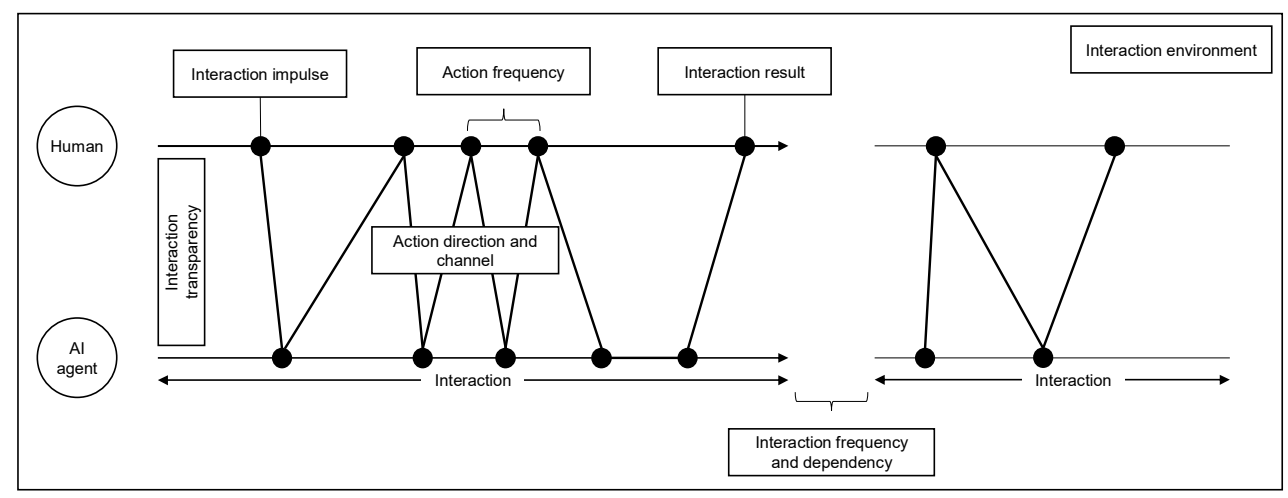

Figure 1. Nine interaction dimensions of a human-Al interaction 
emergency brake assistant during everyday driving. The interaction impulse describes the beginning of a new $\mathrm{H}-\mathrm{AI}$ interaction as well as the reason for it. The model distinguishes between impulses that have a targeted, searching, or play/creative origin. The interaction result describes how an interaction's result influences the environment; it can have monitoring, informing, assisting, advising, or experiential character. The action direction indicates the direction of the individual actions in an interaction. Some interactions contain only actions in one direction, either human to AI agent or vice versa. Interactions with bidirectional actions also exist. The action channel distinguishes the usual sensory forms of perception through which an interaction partner can perceive external stimuli. Information can be transmitted and exchanged acoustically (e.g., via voice commands), optically (e.g., with letters, numbers, and symbols), or haptically (e.g., via vibration, temperature changes, or flexible control elements). The action frequency describes the number of actions in an interaction. When there is only one action in an interaction, this is a simple action frequency; if there are several, this is a multiple-action frequency. The interaction frequency indicates the number of interactions within a certain period. The number of interactions in a specific application ranges from onetime, to rare, to frequent. The interaction dependency describes a measure of the dependency level between one or more interactions. It classifies interactions as either independent (non-interdependent) or interdependent. The interaction environment describes an interaction's current environment. H-AI interactions can be found in both private and professional environments.

\subsection{Human-AI interaction types}

We identified five characteristic interaction types of current or potential AI use cases - guardian angels, pixies, informants, colleagues, and best friends - that describe the characteristics of AI agents when interacting with humans. We introduce the H-AI interaction types in Table 2. We further map the identified interaction types in Figure 2 by considering the characteristics 'freedom of action' and 'reciprocal engagement'. By referring to freedom of action, we

Table 2. Five human-Al interaction types

\begin{tabular}{|c|c|c|c|c|}
\hline & $\begin{array}{r}\text { Description } \\
\end{array}$ & Exemplary expert quotes & Examples & Constituent dimensions \\
\hline 总 & $\begin{array}{l}\text { Guardian angel includes interactions that } \\
\text { are entirely unconscious for persons, up } \\
\text { to their results, and sometimes even } \\
\text { beyond. }\end{array}$ & $\begin{array}{l}\text { "All things that are around us become } \\
\text { more intelligent, so that means more } \\
\text { adaptable. They adapt to certain situations } \\
\text { and we're not even aware of that. So that's } \\
\text { kind of the interaction I don't even notice, } \\
\text { although of course I do interact anyway" } \\
\text { (E25) }\end{array}$ & \begin{tabular}{|l|} 
Vehicle assistance \\
systems such as an \\
emergency braking or \\
lane departure \\
warning system
\end{tabular} & $\begin{array}{l}\text { Interaction impulse: } \\
\text { targeted } \\
\text { Interaction result: } \\
\text { informing, monitoring } \\
\text { Interaction transparency: } \\
\text { unconscious }\end{array}$ \\
\hline בֶ. & $\begin{array}{l}\text { A pixie is an AI agent that mainly } \\
\text { performs basic activities in daily life. } \\
\text { Specifically, a pixie takes over repetitive } \\
\text { tasks to reduce or simplify the human } \\
\text { workload. }\end{array}$ & $\begin{array}{l}\text { "So, it's like a busy little bee, if it's not sure } \\
\text { enough, it'll forward or route it to the right } \\
\text { agent. And if it's sure enough, and that's } \\
\text { usually when it's one of the very repetitive, } \\
\text { very simple requests, then it will sort of } \\
\text { keep that from the support agents and } \\
\text { automate it immediately so that just less } \\
\text { arrives" (E11). }\end{array}$ & $\begin{array}{l}\text { Intelligent software } \\
\text { applications in HR } \\
\text { management such as } \\
\text { CV screening }\end{array}$ & $\begin{array}{l}\text { Interaction impulse: } \\
\text { targeted } \\
\text { Interaction result: } \\
\text { assisting } \\
\text { Interaction transparency: } \\
\text { conscious }\end{array}$ \\
\hline 芯 & $\begin{array}{l}\text { While an informant also takes over basic } \\
\text { activities like a pixie, an informant's } \\
\text { interaction has a stronger focus on } \\
\text { obtaining information. }\end{array}$ & $\begin{array}{l}\text { "If I now have a bot here and it finds the } \\
\text { information I need relatively quickly, } \\
\text { creates tickets for me and is also linked to } \\
\text { the telephone system, then that usually } \\
\text { brings great added value" (E08). }\end{array}$ & $\begin{array}{l}\text { AI service robots } \\
\text { such as shopping or } \\
\text { reception assistance } \\
\text { robots }\end{array}$ & $\begin{array}{l}\text { Interaction impulse: } \\
\text { targeted, searching } \\
\text { Interaction result: } \\
\text { informing } \\
\text { Interaction transparency: } \\
\text { (un)conscious } \\
\end{array}$ \\
\hline 总 & $\begin{array}{l}\text { A colleague is goal-oriented and delivers } \\
\text { an informative, assisting, or consulting } \\
\text { result in conscious interactions. }\end{array}$ & $\begin{array}{l}\text { "For me, it's kind of, it's about assisting } \\
\text { and taking away decisions and it's about } \\
\text { the machines having a perception" (E10). }\end{array}$ & $\begin{array}{l}\text { GPT-3 text creation } \\
\text { software developed } \\
\text { by OpenAI }\end{array}$ & $\begin{array}{l}\text { Interaction impulse: } \\
\text { targeted, searching } \\
\text { Interaction result: } \\
\text { assisting, informing } \\
\text { Interaction transparency: } \\
\text { conscious }\end{array}$ \\
\hline 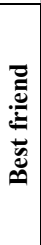 & $\begin{array}{l}\text { A is an interaction type that is } \\
\text { extraordinarily complex owing to its } \\
\text { extensive interdependence. At the same } \\
\text { time, the best friend only interacts within } \\
\text { the pre-defined social action framework. } \\
\text { Interactions with social chatbots involve } \\
\text { consciously experiencing a social } \\
\text { exchange. }\end{array}$ & $\begin{array}{l}\text { "I could certainly imagine that in a private } \\
\text { environment. If you consider the intimate } \\
\text { relationship that some people develop with } \\
\text { game characters in their online worlds, } \\
\text { that can certainly develop into such a } \\
\text { virtual personality that supports them } \\
\text { there" (E05). }\end{array}$ & $\begin{array}{l}\text { Social chatbots such } \\
\text { as Xiaoice, which can } \\
\text { perceive, process, and } \\
\text { create text and speech } \\
\text { with social } \\
\text { competence. }\end{array}$ & $\begin{array}{l}\text { Interaction impulse: } \\
\text { playful, creative } \\
\text { Interaction result: } \\
\text { experiencing } \\
\text { Interaction transparency: } \\
\text { conscious }\end{array}$ \\
\hline
\end{tabular}




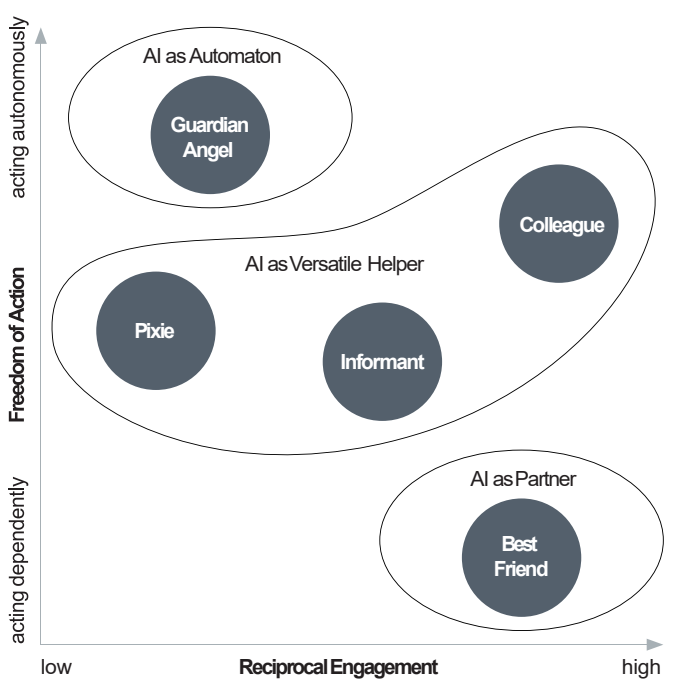

Figure 2. Five human-Al interaction types

acknowledge the AI agents' autonomy as a key characteristic of the AI frontier [24]. We use reciprocal engagement as the second characteristic that emphasizes an H-AI interaction's collaborative nature. Based on these two characteristics, we can group the interaction types in a meaningful way to better inform practice.

We understand freedom of action as the degree of autonomy with which an AI agent can act independently within a given framework. If the freedom of action remains low, an AI agent may only carry out actions dependent on the human actor's impulses; if an AI agent has high freedom of action, it may act autonomously without a concrete human impulse. We define reciprocal engagement between the participants in the interaction as the perception and consideration of the other participant's behavior in an interaction [25]. Thus, the better the reciprocal engagement, the better the interaction's participants understand the mutual expectations and behaviors, allowing them to adapt their actions accordingly. Interaction participants may exchange information or act based on mutual and empathic understanding.

In Figure 2 we see three interaction type groups with similar characteristics: AI agents as an automaton, AI agents as a versatile helper, and AI agents as a partner. The shared use cases from group 1 (AI agents as an automaton) can be described as guardian angels owing to their protective character. Group 2 (AI agents as versatile helper) comprises the three interaction types pixie, informant, and colleague. The use cases in this group have a strong helper character since they support people in their daily lives cooperatively or collaboratively. AI applications' versatility as a versatile helper is reflected in its informative, assisting, and advisory results. In group 3 (AI agents as partner), the interaction type is best friend. This interaction type distinguishes itself from the other groups through its pronounced social and emotional intelligence skills and is thus a companion in daily life.

The findings of Bittner et al. [11] present interaction types which are comparable to group 2 . They present a design taxonomy for conversational agents (CAs) as collaborative teammates whereby they differentiate between the role of CAs as facilitator (comparable to a pixie), peer (comparable to a colleague), and expert (comparable to an informant). However, since we looked beyond collaboration work practices, we could identify two more interaction types based on our empirical data.

\section{How organizations can design purposeful human-AI interactions}

Our interview partners highlight the importance of meeting users' expectations for the H-AI interaction design because it influences users' acceptance and trust. "Expectation management proactively addresses realistic use cases [...] as early as possible and [...] shape and guide expectations with customers" (E08). Beyond the influence of users' expectations on acceptance and trust, we identified transparency, anthropomorphism, and personalization as influencing factors. Their relevance varies depending on the interaction type and the interaction context. In the following, we outline our findings on the influencing factors and their impact on users' acceptance and trust. To make them more tangible and to incorporate them into the design of $\mathrm{H}-\mathrm{AI}$ interactions, we derive three design guidelines per influencing factor. To put the guidelines into context, we depict the usage or lack of these guidelines with examples of AI use cases (see Table 3).

Confirm users' expectations of human-AI interactions to increase acceptance and trust. Technology usage must confirm people's expectations to positively affect their acceptance and trust in technology and to influence their intentions to repeatedly use it [7]. Meeting people's expectations is a challenging task, especially concerning AI, owing to the huge media attention it receives. "I would say that people's expectations of the interface and the usability of certain applications are relatively high. Perhaps due to the media, the reporting, science fiction movies" (E08). Thus, many people have distorted perceptions and expectations of AI's capabilities and performance.

These perceptions and expectations differ in people's private and professional lives. Although many people interact with AI agents in their private lives in playful and fault-tolerant ways, ambivalent expectations 
can often be observed in professional contexts. "From my point of view, that's because in our private lives we do everything that is practical and fun. You immediately have full acceptance. [...] But as soon as it's a question of one's own job or existence, the concern is incredibly great" (E10). Here, humans face AI applications with fears and worries, because they perceive them as a threat that considerably changes or even completely replaces their job [24]. They develop unrealistically high expectations of AI capabilities that cannot yet be confirmed. Thus, interaction outcomes often result in frustration and rejection. "Today I rather have the feeling that the expectations are huge and I am rather disappointed about what comes out in the end" (E25). However, people often neglect the fact that they themselves often perform their tasks erroneously. In cases where errors by AI agents are not tolerated, persons' erroneous behaviors are fully accepted. Skepticism towards AI agents also exists because many people perceive AI agents as a competitor, especially in their professional lives and doubt their self-esteem. The extent to which a person is affected by AI agents is usually determined by their attitude [7]. Thus, considering the potential target users' expectations of and experiences with AI capabilities will help developers to understand which interaction type may best fit the to-be-designed use case, to positively affect users' acceptance and trust. Thus, we define the following design guidelines to better meet users' expectations:

1) Evaluate whether an AI technology is the appropriate technology and fits the expected interaction context.

2) Assess users' experience level with AI technology to design a solution that fits the identified level.

3) Involve users in the design process to identify and address potential needs and worries.

Increase transparency for people to overcome potential resistance. There are two aspects to understanding transparency in the context of $\mathrm{H}-\mathrm{AI}$ interactions. First, transparency refers to humans' awareness of interacting with AI agents. Being aware of an interaction with an AI agent leads to adapted human behaviors [4]. For instance, if humans know they are communicating with a chatbot, they adapt their writing style by using simpler sentences to get the expected results. The same can be observed when people interact with robots. Compared to human-human interaction, people are less expressive in their interactions with a human-like robot than with a person. "So, if you introduce a bot solution like that, then you should really say that it's a bot. This also has other advantages, namely the one advantage that customers simply interact differently or write differently than when they write with a real person" (E11).
Second, transparency refers to the traceability of how AI agents produce their results. Since many people react with fear and resistance to AI agents in their professional lives, they wish to understand how an outcome is generated to get a feeling of control and reliability. "I think in the large population there will continue to be a great distrust of AI developments, simply because power is then there in a form that is not controllable and possibly not understandable" (E05). Thus, implementation methodologies of the explainable AI field support the comprehensibility of AI agents without negatively influencing their performance. The secure feeling of better understanding AI agents' functionalities and outcomes helps people to interact more effectively with them and to build acceptance and trust. "I think it's critical, that as the systems get implemented, they can explain how they derive the decision. So, explainability is important in some cases, not in all cases" (E04).

We can observe a difference in the desire to understand the AI frontier in people's private lives. Here, it rarely adds value for them to know when humans interact with AI agents or how an outcome is generated. People want to ease their lives by substituting their daily activities, leading to a pleasurable feeling. The desired result is in focus, delivered by a technology that operates in the background as far as possible. Humans do not have to consciously perceive or understand an AI agent or the technology behind it to experience its added value. For instance, very few people know or even wonder how exactly a driving assistant works, just as today we no longer question a television's functioning or benefits. "Humans do not see AI per se, they do not need to see it or even know what is happening. Humans have an application, like the vehicle is now parking." (E15) Thus, if use cases are created for people's private lives in which either AI agents act as a guardian angel independently in the background, or directly in the role of a best friend, designers do not have to incorporate features to reveal how an interaction outcome is produced since people are open to accept and trust AI agents in this context.

However, even if the demand for transparency exists, it often cannot be implemented. A complete understanding of $\mathrm{AI}$ agents is often too complex, or the underlying methods and algorithms allow only limited insights [10]. It is only necessary in certain cases because we can use and accept technologies without knowing every technical detail - as has already been shown by telephones, radios, or televisions. Thus, a key success factor is expectation management i.e., present the capabilities and limitations of an AI agent in a sufficiently transparent way. What an AI agent achieves, how, and in what result quality is important information that increases people's understanding and determines $\mathrm{H}$ - 
AI interactions' success. The appropriate degree of transparency varies depending on the initial situation and the interaction context. Consequently, we derive design guidelines for implementing an appropriate degree of transparency:

1) Examine what degree of transparency is appropriate for the expected interaction context (private vs. professional life) to increase users' acceptance of and trust in AI agents.

2) Incorporate features to reveal the input parameters and the underlying dataset to facilitate users' understanding of the created interaction outcome, preferably in the professional context.

3) Incorporate human-like interaction patterns to overcome potential user resistance against transparency gaps in created interaction outcomes.

Choose a reasonable degree of a human-like appearance. A human-like behavior and appearance may ease $\mathrm{H}-\mathrm{AI}$ interaction because it can invoke rules and norms of human social interaction [26]. However, anthropomorphism is an ambivalent design variable that can positively or negatively impact user experience, depending on the interaction context and the end-user characteristics. On the one hand, human-likeness increases users' engagement in the interaction and perception of social cues and presence [4]. Especially, the increased perceived social presence influences a user in interactions with a human-like AI agent, since it can positively impact the interaction enjoyment as well as the perceived usefulness of and the trust in the AI agent [5]. The perceived social presence creates a higher usage intention and increases user compliance. In contrast, it is not necessary to pretend real human involvement but to be transparent that the user interacts with an AI agent. On the other hand, human-likeliness can also negatively influence an interaction when emerging expectations from human-like behavior are not met [12]. Depending on the interaction context, human-like features are more or less required. If AI agents act as a guardian angel to protect people, or as an informant to provide information, a less human-like appearance may be necessary than acting like a best friend with more social and emotional characteristics as well as a higher engagement in the interaction from both parties.

Anthropomorphism directly influences people's expectations of AI capabilities and their interactions with AI agents. "I think when you make something have human characteristics, you have to some degree human expectations to it and Alexa is far from human and has a very limited range of capabilities" (E03). If these result in excessive expectations, there is a risk that they will not be met, resulting in disappointment, frustration, and - ultimately - rejection. Creating human-like and natural effects also builds an additional inhibition threshold, since it increases people's fears of becoming superfluous. Interaction is often about appreciation and self-confidence. Thus, these two factors will suffer additionally if a person has the impression that a machine may do their job better. Therefore, we specify design guidelines to incorporate human-like behavior and appearance usefully:

1) Check for available technical and contextappropriate human-like features.

2) Evaluate the potential benefits of using human-like features and if users will appreciate them in the expected interaction context.

3) Appraise whether chosen human-like features can meet related users' expectations and can disclose whether features will not work as in a comparable human-human interaction.

Enable personalization of AI applications to users' preferences to facilitate interactions. Personalization affects users' attitudes toward AI agents by observing user preferences, collecting user data, and considering users' chosen default settings [27]. Thus, AI agents consider users' preferences in an action-oriented way to tailor interactions. The more AI agents are personalized, the more individually they interact with a user so that, for instance, the user only receives relevant interaction results and content. Thus, highly personalized AI agents could reduce interaction frequency, so that users must interact as rarely as possible. As the AI agent interacts with users based on their individual preferences, users feel understood and consider their motives as fulfilled. Having these positive experiences, an AI agent successfully meets users' expectations resulting in increased acceptance and trust [28].

However, personalization can also negatively influence interactions. By collecting personal user data without users' awareness, they may be surprised that they are interacting with an AI agent by receiving unexpected interaction outcomes. "And if the system learns that automatically, you feel that the system knows perhaps a little too much and can make inferences. Now, the inference is useful to you, but the background by which it derived that inference is disturbing" (E04). Such negative system experiences can lead to rejection of an AI agent and can destroy acceptance and trust as users feel that they have lost control over the system [29]. It is crucial to make users aware of the learning process of AI agents based on personal data, in the best case by enabling active influencing of the learning process. The personalization of an AI agent develops further via interactions with users and therefore represents a dynamic influencing factor.

Depending on the role an AI agent takes on, more personalization is required. The higher the reciprocal engagement between people and AI agents, the more personalization features are required to enable an AI agent to react to people's individual needs, such as if an 
$\mathrm{AI}$ agent acts as a best friend. In this context, people may more easily accept the collection of personal data because they know that they are interacting with an AI agent, and they expect it to interact with them individually. Thus, we define guidelines to implement personalization features to ease the interaction for users:

1) Evaluate the available personalization features and the appropriate degrees for the interaction context.

2) Reveal which personal user and context-related data are collected and processed.

3) Enable users to control the use of personal data individually and dynamically, even if this may lead to reduced functionality.

A purposeful design helps to build acceptance and trust in human-AI interactions. Successful H-AI interactions require purposeful design if they are to meet users' expectations. Acceptance of new technologies is a critical success factor for their adoption, and we must distinguish between acceptance after initial use (preacceptance) and the intention to continue using (postacceptance) [7]. Pre-acceptance can be achieved by considering the ease of use and the perceived usefulness; both are known from established acceptance models, such as the Technology Acceptance Model (TAM) [30]. Even if ease-of-use is relevant for preacceptance, this is not the case for post-acceptance. Beyond perceived usefulness, users' satisfaction with a technology determines their intention to continue using by confirming users' expectations.

We cannot expect people to fully accept an AI application after a single interaction; rather, people accept AI applications in an iterative process that consists of many interactions. "It is smarter if the machine, which in principle could control itself, first makes suggestions, and the employee can then accept the suggestions or not. And if you then log a little bit where the suggestions were good and could have led to better results, then the employee who was previously responsible for this also gains trust" (E10). It is important to know and manage expectations from the outset. If people's expectations are continually confirmed over time, which facilitates the gradual achievement of high acceptance, users gain more trust in AI agents. Thereby, users increasingly rely on correct results. This is especially important in use cases where AI agents gradually substitute people. The choice of degrees of transparency, anthropomorphism, and personalization is crucial. Although these influencing factors promote a continued successful interaction, they can also manipulate users. Especially the use of a human-like appearance provides this potential by simulating a social presence to influence users' behaviors. Research has shown that human-like features of AI agents may significantly influence important user

Table 3. Examples for design guidelines

\begin{tabular}{|c|c|c|c|}
\hline & Example & Description & Guideline in context (guideline reference) \\
\hline 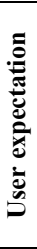 & $\begin{array}{l}\text { Vara breast cancer screening } \\
\text { platform } \\
\text { (https://www.varahealthcare.com/) }\end{array}$ & $\begin{array}{l}\text { AI application to support radiologists by ruling } \\
\text { out normal exams to help them to focus on } \\
\text { potentially suspicious exams } \\
\text { The healthcare context poses specific challenges } \\
\text { owing to sensitive doctor-patient interactions } \\
\text { and users being highly qualified medical } \\
\text { practitioners }\end{array}$ & $\begin{array}{l}\text { Designers involve users in further } \\
\text { development by partnering with them, } \\
\text { receiving new datasets, and including them in } \\
\text { the dataset labeling process (3) } \\
\text { - The active user involvement in the data } \\
\text { quality improvement helps to produce an } \\
\text { outcome that meets the needs of doctors and } \\
\text { patients (3) }\end{array}$ \\
\hline 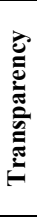 & $\begin{array}{l}\text { Amazon AI recruiting tool } \\
\text { (https://reut.rs } / 2 O d 9 f P r)\end{array}$ & $\begin{array}{l}\text { - A solution to review applicants' résumés to } \\
\text { identify the top five applicants }\end{array}$ & $\begin{array}{l}\text { - Although the underlying data input was clear } \\
\text { (received résumés of the past } 10 \text { years), the } \\
\text { decision-making process and its results } \\
\text { lacked transparency }(1,2) \\
\text { - The data processing led to a bias against } \\
\text { women in the recruiting process, requiring } \\
\text { that the recruiting tool be discontinued }(1,2)\end{array}$ \\
\hline 音 & $\begin{array}{l}\text { CRTL Human } \\
\text { (https://www.quantumcapture.com } \\
\text { /ctrl-human) }\end{array}$ & $\begin{array}{l}\text { A solution to embody conversational AI with } \\
\text { photo-real, interactive avatars capable of two- } \\
\text { way conversation and dynamic body gestures } \\
\text { The solution provides a human-like feature for } \\
\text { enterprises that developed their proprietary } \\
\text { chatbot solution to ease interactions with their } \\
\text { customers }\end{array}$ & $\begin{array}{l}\text { By using a high degree of human-like } \\
\text { features, the avatars simulate social presence, } \\
\text { which seeks to positively influence users' } \\
\text { engagement and enjoyment of interactions } \\
\text { (1-3) }\end{array}$ \\
\hline : & $\begin{array}{l}\text { Xiaoice - Microsoft's China-based } \\
\text { chatbot } \\
\text { (https://www.msxiaobing.com/) }\end{array}$ & $\begin{array}{l}\text { - An AI application to relate to and interact with } \\
\text { people through nuance, social skills, and } \\
\text { emotions } \\
\text { - Xiaoice seeks an appropriate degree of } \\
\text { personalization by learning with each human } \\
\text { interaction, so as to acquire human social skills } \\
\text { based on deep learning techniques }\end{array}$ & $\begin{array}{l}\text { Owing to its personalization features, Xiaoice } \\
\text { has become a friend and trusted confidant of } \\
\text { millions of users (1) }\end{array}$ \\
\hline
\end{tabular}


decisions, such as increased investment volumes in financial portfolio decisions [29]. Thus, designers must consciously choose human-like features to not risk users' acceptance and trust as well as to not interfere with users' intrinsic usage intentions.

Increased acceptance of and trust in AI agents affect users' expectations as they will adjust them based on the newly gained information and experiences [7]. In turn, this influences the subsequent interaction processes. For instance, it is conceivable that a person initially exercises strong control and only uses individual functionalities of an AI agent. In this phase, acceptance and trust increase as the AI agent confirms expectations and successfully facilitates or substitutes individual activities. Thus, the likelihood of experiencing the same benefits in subsequent interaction processes increases, as well as that the AI agent constantly delivers the experienced result quality level. If AI agents are increasingly accepted and trusted, users will be able to make more frequent and extensive use of them. This gives people opportunities to perceive completely new interaction types and to reduce their own actions while, simultaneously, AI agents gain freedom of action. Just as trust slowly builds through accepted interactions, individual interactions in which AI agents do not confirm expectations can destroy already gained trust.

Further, a person's character also determines how they encounter AI agents, from affine, curious, and skeptical, to resistant. "I would rather allude to things like an affinity for technology, curiosity, things like that" (E09). Every person, whatever their age, has different attitudes towards and expectations of technology generally and AI in particular. Even geographical and cultural dependencies can be identified here. While in China new technologies enjoy high acceptance and constant curiosity, a traditionally more skeptical attitude can be observed in Germany, where technologies are considered more critically, and aspects such as security and data protection are in the foreground. Thus, acceptance and adaptation speed also depend on social aspects and the state's view of technology. Anyone who designs use cases that targets successful H-AI interactions should understand and address expectations at an early stage via appropriate expectation management. For instance, fears, worries, and unrealistically high expectations can be considered from the outset, and a realistic idea of what is possible now and, in the future, can be formed.

\section{Conclusion}

With the vast technological development in the near future, AI applications will gradually find their way into our private and professional lives. Vast technological development will enable interaction types that we cannot yet imagine. However, the rapid enhancements will require organizations and society to transition as smoothly as possible into a world where AI agents will be omnipresent. Thus, the purposeful design of AI use cases will become a critical success factor to foster positive attitudes toward AI agents, so that it will no longer be humans against AI agents (us vs. them), but a symbiotic relationship. This development will help organizations exploit the benefits of successful H-AI interactions that are greater than their individual contributions [31]. In the future, humans and AI agents will extend one another's capabilities and competencies in different interaction contexts and will build an augmented intelligence [32]. Drawing on our findings on interaction dimensions, interaction types, and design guidelines as a starting point for future research, designers and developers may benefit from a better understanding of how to design $\mathrm{H}-\mathrm{AI}$ interactions that foster people's acceptance of and trust in AI agents.

In the following, we discuss our studies' limitations and present opportunities for further research. First, the focus of this paper is on presenting and analyzing existing and potential AI-enabled systems as well as emerging $\mathrm{H}-\mathrm{AI}$ interactions from a practical perspective. Thus, the design guidelines we derived only build a first foundation that should be complemented by existing approaches from systems design, UI/UX, and related domains. Second, our research is not designoriented with the objective of developing a useful artifact. Future research can work in this direction in close collaboration with practice. For that purpose, the identified interaction dimensions and characteristics could be of particular importance. Third, our work stimulates $\mathrm{H}-\mathrm{AI}$ interactions as an important perspective for the ongoing debate to theorize AI agents' impact on our personal and work lives. In this context, we see a necessity to embed research on $\mathrm{H}-\mathrm{AI}$ interactions in existing adoption theories and to challenge these theories for eventual AI specifics. Apart from that, a better understanding of $\mathrm{H}-\mathrm{AI}$ interactions helps future research to better understand more realistic and promising scenarios to utilize AI agents in the corporate context - AI agents augmenting humans towards a hybrid intelligence, instead of replacing humans [33].

\section{References}

[1] Brynjolfsson, E. and A. McAfee, "The Business of Artificial Intelligence", Harvard Business Review(July), 2017, 3-11.

[2] Hofmann, P., J. Jöhnk, D. Protschky, and N. Urbach, "Developing Purposeful AI Use Cases - A Structured Method and Its Application in Project Management", in WI 2020 Proceedings, Potsdam, Germany. 2020.

[3] Dellermann, D., A. Calma, N. Lipusch, T. Weber, S. Weigel, and P. Ebel, "The Future of Human-AI 
Collaboration: A Taxonomy of Design Knowledge for Hybrid Intelligence Systems", in HICSS 2019

Proceedings, Maui, Hawaii, USA. 2019.

[4] Rzepka, C. and B. Berger, "User Interaction with AIenabled Systems: A Systematic Review of IS Research", ICIS 2018 Proceedings, 2018.

[5] Adam, M., M. Wessel, and A. Benlian, "AI-based chatbots in customer service and their effects on user compliance", 2020.

[6] Bawack, R.E., S.F. Wamba, and K. Carillo, "Artificial Intelligence in Practice: Implications for IS Research", AMCIS 2019 Proceedings, 2019.

[7] Bhattacherjee, A., "Understanding Information Systems Continuance: An Expectation-Confirmation Model", Management Information Systems Quarterly, 25(3), 2001.

[8] Diederich, S., A.B. Brendel, S. Morana, and L. Kolbe, "On the Design of and Interaction with Conversational Agents: An Organizing and Assessing Review of Human-Computer Interaction Research", Journal of the Association for Information Systems, January, 2022.

[9] Maedche, A., C. Legner, A. Benlian, B. Berger, H. Gimpel, T. Hess, O. Hinz, S. Morana, and M. Söllner, "AI-Based Digital Assistants: Opportunities, Threats, and Research Perspectives", Bus. Inf. Syst. Eng., 61(4), 2019, pp. 535-544.

[10] Rahwan, I., M. Cebrian, N. Obradovich, J. Bongard, J.F. Bonnefon, C. Breazeal, J.W. Crandall, N.A. Christakis, I.D. Couzin, M.O. Jackson, N.R. Jennings, E. Kamar, I.M. Kloumann, H. Larochelle, D. Lazer, R. McElreath, A. Mislove, D.C. Parkes, A.'. Pentland, M.E. Roberts, A. Shariff, J.B. Tenenbaum, and M. Wellman, "Machine behaviour", Nature, 568(7753), 2019, pp. 477-486.

[11] Bittner, E., S. Oeste-Reiß, and J.M. Leimeister, "Where is the Bot in our Team? Toward a Taxonomy of Design Option Combinations for Conversational Agents in Collaborative Work", in HICSS 2019 Proceedings, Maui, Hawaii, USA. 2019.

[12] Pfeuffer, N., A. Benlian, H. Gimpel, and O. Hinz, "Anthropomorphic Information Systems", Bus. Inf. Syst. Eng., 61(4), 2019, pp. 523-533.

[13] Newcomb, T.M., R.H. Turner, and P.E. Converse, Social Psychology, Psychology Press, 2015.

[14] Mortensen, C.D., Communication: The Study of Human Interaction, McGraw-Hill, New York, 1972.

[15] Li, L. and P. Zhang, "The Intellectual Development of Human-Computer Interaction Research: A Critical Assessment of the MIS Literature (1990-2002)", Journal of the Association for Information Systems, 6(11), 2005, pp. 227-292.

[16] Zhang, P. and N. Li, "An assessment of humancomputer interaction research in management information systems: topics and methods", Computers in Human Behavior, 20(2), 2004, pp. 125-147.

[17] Zhang, P., N. Li, M. Scialdone, and J. Carey, "The Intellectual Advancement of Human-Computer Interaction Research: A Critical Assessment of the MIS Literature (1990-2008)", AIS Transactions on HumanComputer Interaction, 1(3), 2009, pp. 55-107.
[18] Hoc, J.M., "From human-machine interaction to humanmachine cooperation", Ergonomics, 43(7), 2000, pp. 833-843.

[19] Honig, S. and T. Oron-Gilad, "Understanding and Resolving Failures in Human-Robot Interaction: Literature Review and Model Development", Frontiers in Psychology, 9, 2018, p. 861.

[20] Jöhnk, J., M. Weißert, and K. Wyrtki, "Ready or Not, AI Comes - An Interview Study of Organizational AI Readiness Factors", Bus. Inf. Syst. Eng., 63(1), 2021, pp. 5-20.

[21] Arksey, H. and L. O'Malley, "Scoping studies: towards a methodological framework", International Journal of Social Research Methodology, 8(1), 2005, pp. 19-32.

[22] Myers, M.D. and M. Newman, "The qualitative interview in IS research: Examining the craft", Information and Organization, 17(1), 2007, pp. 2-26.

[23] Saldaña, J., The coding manual for qualitative researchers, 2nd edn., Sage Publications, Los Angeles, 2013.

[24] Nicholas Berente, Bin Gu, Jan Recker, and Radhika Santhanam, "Managing Artificial Intelligence", MIS Quarterly, 45(3), 2021, pp. 1433-1450.

[25] Sidner, C.L., C.D. Kidd, C. Lee, and N. Lesh, "Where to look", in International Conference on Intelligent User Interfaces Proceedings, Funchal, Madeira, Portugal. 2004.

[26] Hinds, P., T. Roberts, and H. Jones, "Whose Job Is It Anyway? A Study of Human-Robot Interaction in a Collaborative Task", Human-Computer Interaction, 19(1), 2004, pp. 151-181.

[27] Fan, H. and M.S. Poole, "What Is Personalization? Perspectives on the Design and Implementation of Personalization in Information Systems", Journal of Organizational Computing and Electronic Commerce, 16(3-4), 2006, pp. 179-202.

[28] Sun, J., "Ubiqutious System Capabilities and User Readiness: An Activity Perspective", PACIS 2016 Proceedings, 2016.

[29] Adam, M., J. Toutaoui, N. Pfeuffer, and O. Hinz, "Investment Decisions with Robo-Advisors: The Role of Anthropomorphism and Personalized Anchors in Recommendations", in ECIS 2019 Proceedings, Stockholm \& Uppsala, Sweden. 2019.

[30] Davis, F.D., R.P. Bagozzi, and P.R. Warshaw, "User Acceptance of Computer Technology: A Comparison of Two Theoretical Models", Management Science, 35(8), 1989, pp. 982-1003.

[31] Xu, B., X. Song, Z. Cai, A. Chong, E. Lim, C.-W. Tan, and $\mathrm{J}$. Yu, "Artificial Intelligence or Augmented Intelligence: A Case Study of Human-AI Collaboration in Operational Decision Making", PACIS 2020 Proceedings, 2020.

[32] Rui, Y., "From Artificial Intelligence to Augmented Intelligence", IEEE MultiMedia, 24(1), 2017, pp. 4-5.

[33] Dellermann, D., P. Ebel, J.M. Leimeister, and M. Söllner, "Hybrid Intelligence", Bus. Inf. Syst. Eng., 61(5), 2019, pp. 637-643. 